\title{
Changes in the endogenous BMP expression during redifferentiation of chondrocytes in 3D cultures
}

\author{
CHRISTIAN ALBRECHT ${ }^{1}$, WERNER SCHLEGEL ${ }^{2}$, PHILIPP BARTKO $^{3}$, PETER ECKL $^{4}$, \\ TAMARA JAGERSBERGER ${ }^{1}$, VILMOS VÉCSEI ${ }^{1}$ and STEFAN MARLOVITS ${ }^{1}$ \\ ${ }^{1}$ Department of Traumatology, Center for Joint and Cartilage, Austrian Cluster for Tissue Regeneration, and \\ Departments of ${ }^{2}$ Paediatrics, ${ }^{3}$ Internal Medicine, Medical University of Vienna, Waehringer Guertel 18-20, \\ 1090 Vienna; ${ }^{4}$ Department of Cell Biology, University of Salzburg, Hellbrunner Strasse 34, 5020 Salzburg, Austria
}

Received March 19, 2010; Accepted May 17, 2010

DOI: 10.3892/ijmm_00000468

\begin{abstract}
Engineering cartilage tissue is challenging, mainly because chondrocytes lose their differentiated phenotype when cultured in monolayer. The aim of this study was to analyse the influence of 3D-culture conditions on the redifferentiation of chondrocytes, devoting special attention to BMPs. Dedifferentiated chondrocytes were seeded onto two different scaffolds (Bio-Gide ${ }^{\circledR}$ and HYAFF-1 $1^{\circledR}$ ) and were then cultured for 38 days. Every week, samples were taken for gene expression analysis and immunohistochemistry. In both scaffolds an increasing differentiation was observed caused by an increase in Col2 and a reduction in Coll expression. The various BMPs were regulated, albeit differently by the changing culture conditions. While $G D F-5$ and $B M P-4$ expression increased in the monolayer culture in comparison with native cartilage and decreased again in the $3 \mathrm{D}$ culture, the $B M P-2$ and $B M P-6$ expression decreased dramatically in the monolayer as well as in the $3 \mathrm{D}$ culture. $B M P-7$ was not detectable in any probe. Scaffold cultivation appears to stimulate the induction of redifferentiation, but is not sufficient to induce expression of $B M P-2$-6 or -7 . Since, in comparison to cartilage development, there is a lack of surrounding signal centres, external stimuli seem to be required to obtain complete redifferentiation. Our data indicate that a combination of BMP-2, -6 and -7 may be promising for this purpose.
\end{abstract}

\section{Introduction}

Articular cartilage is a promising target for tissue regeneration strategies since cartilage defects have a bad regeneration capacity and cause osteoarthritis in the long-term (1). This is

Correspondence to: Dr Christian Albrecht, Department of Traumatology, Center for Joint and Cartilage, Medical University of Vienna, Waehringer Guertel 18-20, 1090 Vienna, Austria E-mail: christian.albrecht@meduniwien.ac.at

Key words: chondrocytes, cartilage, BMP, redifferentiation responsible for large socioeconomic problems. Furthermore, specific properties, e.g., the existence of only one cell type or the lack of vascularisation and innervation, make cartilage an ideal candidate for tissue engineering. The process of cartilage tissue engineering has, however, turned out to be very challenging.

One cell-based method, which has already been in clinical use for several years, is called matrix-associated chondrocyte transplantation (MACT). This two-step technique requires articular chondrocytes to be isolated and propagated in monolayer cultures. During cultivation in monolayer, the cells undergo morphological and molecular-biological changes. This process is called dedifferentiation $(2,3)$. The cells lose their rounded cell shape, become elongated and decrease the synthesis of collagen type II in favour of collagen type I (4-6). In order to obtain differentiated cells for transplantation, several methods have been established to induce redifferentiation after cell propagation. The most common method is the use of 3D-culture conditions. A variety of different materials are used as scaffolds, including collagen I/III membranes (7), hyaluronic fleeces (8), collagen gels (9) and collagen matrices (10). Other procedures that can be used alone or in combination with $3 \mathrm{D}$-culture conditions include the supplementation of differentiation factors, mechanical stimulation of the cells and the use of low oxygen tension during cultivation (11-13).

One family of proteins that is promising for cartilage redifferentiation are the bone morphogenetic proteins (BMPs). BMPs are a subfamily of the TGF- $\beta$ superfamily and consist of approximately 20 members (14). They are involved in embryonic development and tissue homeostasis and govern multiple functions, such as differentiation and cell proliferation. They signal through serine/threonine kinase receptors (BMPRs), themselves composed of type I and II subtypes $(15,16)$. To date three type I receptors (BMPR-Ia, BMPR-Ib and ActR-Ia) and 3 type II receptors (BMPR-II, ActR-II and ActR-IIb) have been identified (17-21). Ligand binding leads to the assembly of a tetrameric complex of BMPRs, consisting of two type I and two type II receptors (22). Signal transfer is obtained by Smad-1, -5 and -8 , which are phosphorylated by the receptor complex. After phosphorylation, the smad proteins associate with Smad-4. This complex is translocated 
into the nucleus and evokes alterations in the transcription of different genes (14).

In several studies it has been demonstrated that BMPs [including BMP-2, BMP-4, BMP-6, BMP-7 and growth and differentiation factor 5 (GDF-5 or BMP-14)] have a positive effect on the differentiation of mesenchymal stem cells towards chondrocytes (23-25) as well as on the redifferentiation of chondrocytes (26-30). Very little is known, however, about the endogenous expression of BMPs during redifferentiation of chondrocytes, although this should have an impact on the selection of the appropriate BMPs added during cultivation. Therefore, our aim was to analyse the redifferentiation behaviour of chondrocytes cultured on two different scaffolds with special attention devoted to the expression of BMPs.

\section{Materials and methods}

Isolation and monolayer culture of human articular chondrocytes. Human articular cartilage samples were collected from the hips of four patients (aged 43-81 years) with no history of joint disease, who were scheduled to undergo joint replacement following femoral neck fracture (approved by the Ethics Board of the University of Vienna; code: 184/98). Noncalcified cartilage was dissected from the bone immediately after surgery. The cells were isolated and cultured in monolayer according to our standard operation procedure, as previously described (5). From the same cartilage used for the cell culture a fraction was pooled and used as native cartilage control. Cells isolated from the ligamentum capitis femoris were used as a fibroblast control. The chondrocytes were dedifferentiated in monolayer culture for six weeks and were seeded on two scaffolds $\left(1 \times 10^{6}\right.$ cells $\left./ \mathrm{cm}^{2}\right)$ consisting of hyaluronan (HYAFF- $11^{\circledR}$, Fidia, FAB, Italy) or porcine collagen I/III (Bio-Gide ${ }^{\circledR}$, Geistlich Pharma, Wolhusen, Switzerland) for redifferentiation. The culture medium for both the monolayer culture and the $3 \mathrm{D}$ culture consisted of DMEM, containing 10\% fetal calf serum, 2 g/l HEPES, $1 \%$ L-glutamine, $100 \mu \mathrm{g} / \mathrm{ml}$ streptomycin, $2.5 \mu \mathrm{g} / \mathrm{ml}$ fungizone (all from Life Technologies) and $50 \mathrm{mg} / \mathrm{l}$ ascorbic acid (Sigma-Aldrich, St. Louis, MO).

RNA extraction and purification. Total RNA was obtained from cells cultured in monolayer and on 3D scaffolds. Cells were harvested by adding $1 \mathrm{ml}$ of TRI reagent ${ }^{\mathrm{TM}}$ (SigmaAldrich). Lysis of the cells was performed directly on the culture dish. RNA isolation was performed according to the standard protocol.

For total RNA extraction from native cartilage, slices of human articular cartilage were frozen in liquid nitrogen and ground using a mortar and pestle. Further steps were performed using the RNeasy ${ }^{\circledR}$ plant mini kit (Qiagen, Germany). The procedure for isolating total RNA was executed as described in the RNeasy mini handbook (06/2001, Qiagen).

cDNA synthesis. Total RNA $(0.1-1 \mu \mathrm{g})$ was diluted with nuclease-free water to a volume of $15 \mu 1$. Thereafter $4 \mu 1$ iScript $^{\mathrm{TM}}$ reaction mix, as well as $1 \mu 1$ iScript reverse transcriptase were added (Bio-Rad Laboratories, Hercules,
Table I. Description of the designed primers and probes.

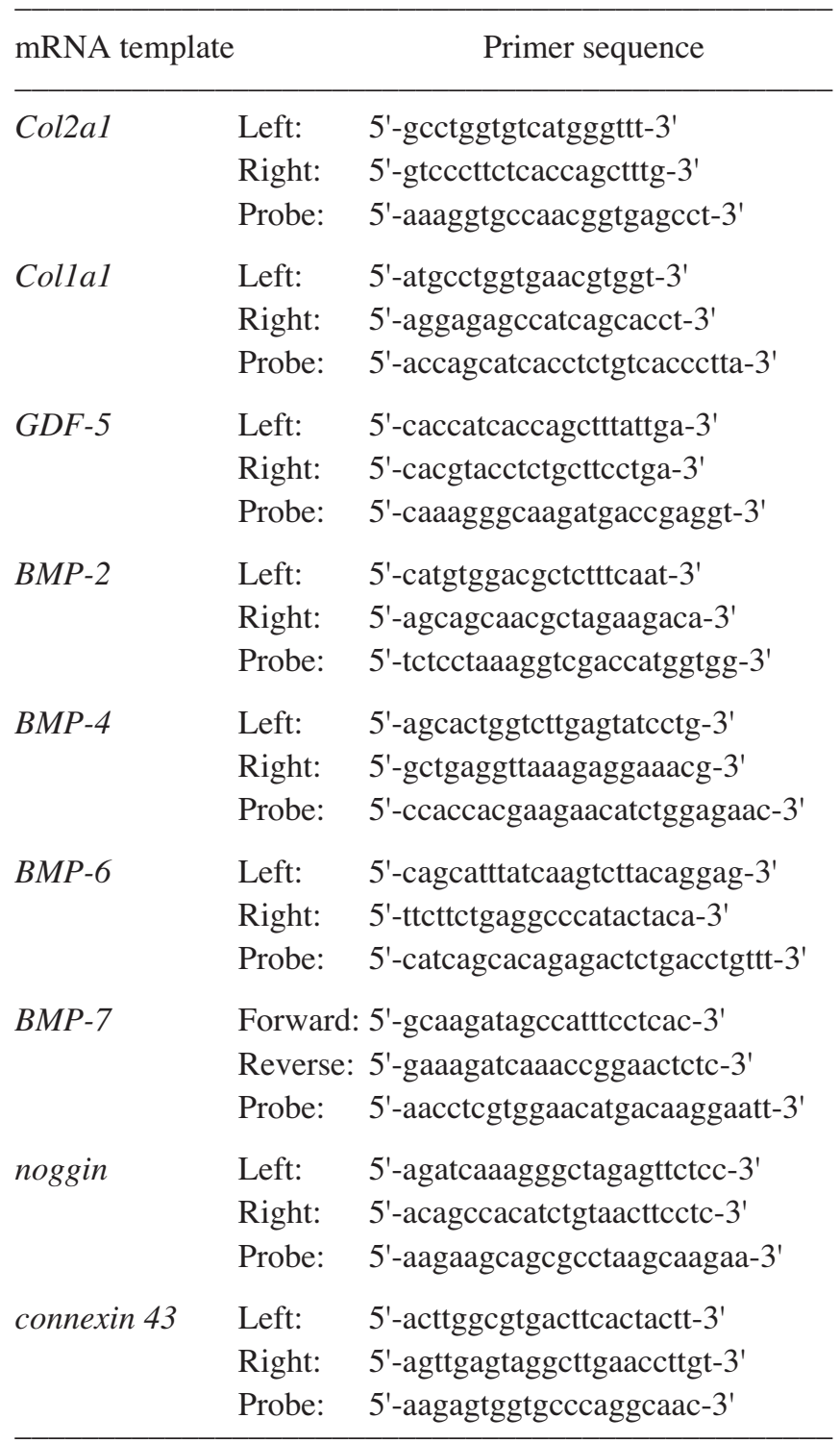

CA, USA). The reaction mixture was incubated for $5 \mathrm{~min}$ at $25^{\circ} \mathrm{C}$, for $30 \mathrm{~min}$ at $40^{\circ} \mathrm{C}$, and for $5 \mathrm{~min}$ at $85^{\circ} \mathrm{C}$.

Primers and probes for quantitative analyses. Primers and probes were designed using the Primer3 program (http:// frodo.wi.mit.edu/cgi-bin/primer3/primer3_www.cgi) which generates oligonucleotides with similar melting temperatures and minimal self complementarity. To avoid amplification of genomic DNA, the probes were placed at the junction of two exons. Gene specificity of the primers and probes and the absence of DNA polymorphism were confirmed by BLAST searches. Primers and probes were synthesised by biomers.net (Ulm, Germany). Primer concentrations were tested for each primer at concentrations of 50, 300 and $900 \mathrm{nM}$, choosing the combination that displayed the lowest $\mathrm{Ct}$ value. Primer sequences are listed in Table I.

Real-time PCR amplification and analysis. Real-time PCR amplification was performed and monitored using an ABI PRISM $^{\circledR} 7500$ Fast real-time PCR system (Perkin-Elmer 

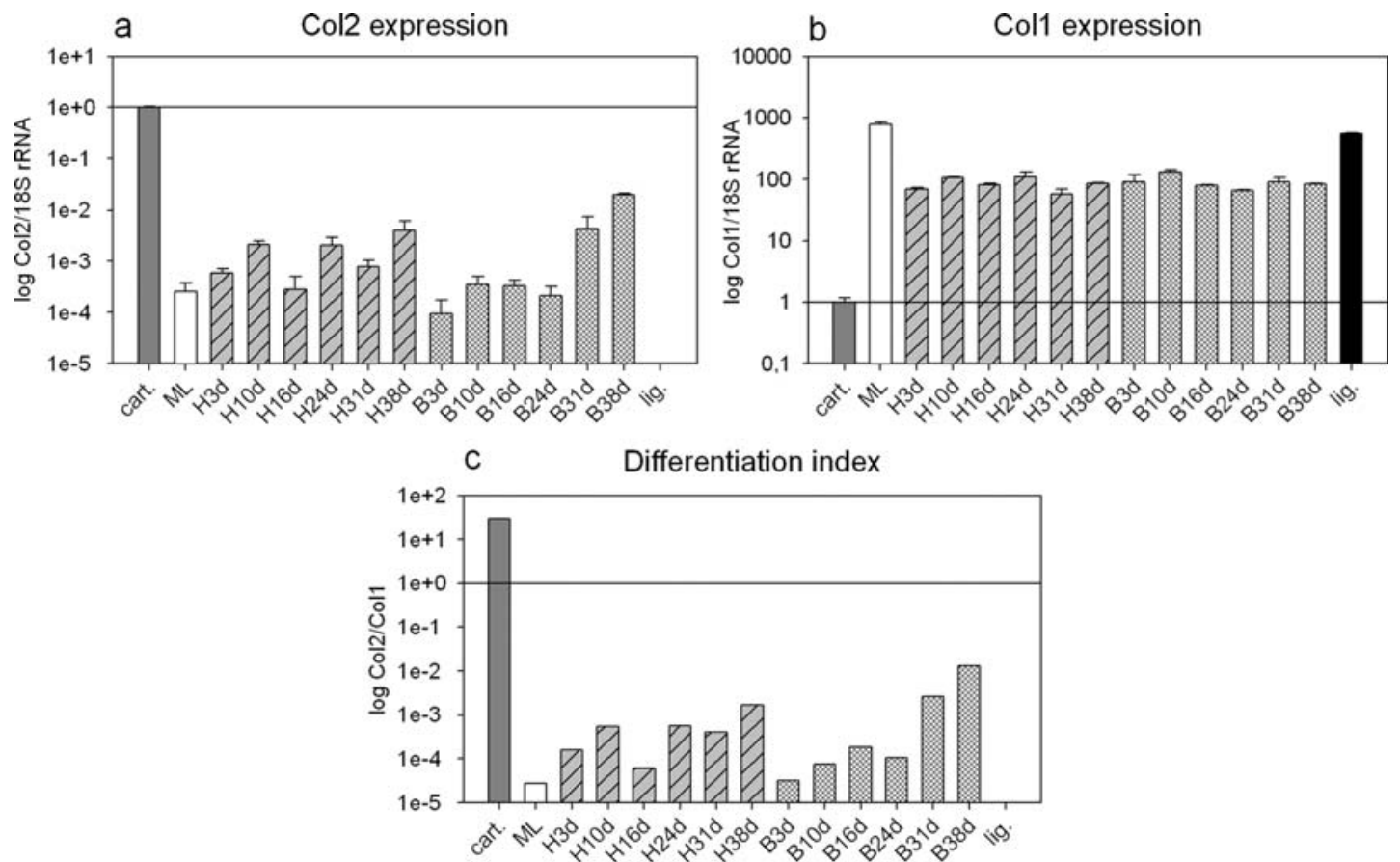

Figure 1. Real-time PCR analysis of Col2 and Col1 expression in native cartilage (cart.), dedifferentiated chondrocytes which were cultured for 6 weeks in monolayer (ML), dedifferentiated chondrocytes seeded on a HYAFF-11 fleece (H3d-H38d) or a Bio-Gide membrane and cultured for 3-38 days (B3d-B38d) and ligament fibroblasts (lig.). Gene expression was normalized to $18 S \mathrm{rRNA}$ expression. Data were log transformed and expressed as mean \pm SD of the realtime PCR triplicate analysis. The dedifferentiation index was calculated as the ratio of the Col2 expression divided by the Coll expression.

Applied Biosystems, Foster City, CA, USA). The master mix was based on the Brilliant ${ }^{\mathrm{TM}}$ QPCR master mix (Stratagene, San Diego, CA, USA). The thermal cycling conditions comprised the initial steps at $50^{\circ} \mathrm{C}$ for 2 min and at $95^{\circ} \mathrm{C}$ for $10 \mathrm{~min}$. Amplification of the cDNA products was performed with 40 PCR cycles, consisting of a denaturation step at $95^{\circ} \mathrm{C}$ for $15 \mathrm{sec}$ and an extension step at $60^{\circ} \mathrm{C}$ for $1 \mathrm{~min}$. For SYBR Green amplification, an additional dissociation step $\left(95^{\circ} \mathrm{C}\right.$ for $15 \mathrm{sec}, 60^{\circ} \mathrm{C}$ for $20 \mathrm{~min}$ and $95^{\circ} \mathrm{C}$ for $15 \mathrm{sec}$ ) was performed. $18 S$ rRNA was chosen as the internal standard, using the predeveloped Taq Man ${ }^{\circledR}$ assay (Applied Biosystems). All cDNA samples ( $3 \mu 1$ in $25 \mu \mathrm{l}$ ) were analysed in triplicate. The final numeric value was calculated as the ratio of the gene to $18 S$ rRNA and was expressed in arbitrary units.

Histology and immunohistochemistry. For histological examinations standard protocols were used. In brief, HYAFF-11 and Bio-Gide membranes were fixed with $4 \%$ paraformaldehyde, dehydrated in alcohol, rinsed in xylol and infiltrated with paraffin. Deparaffinized sections were permeabilized with $20 \mu \mathrm{g} / \mathrm{ml}$ Proteinase $\mathrm{K}$ for $15 \mathrm{~min}$ at $37^{\circ} \mathrm{C}$. After incubation with Image-iT FX Signal Enhancer (Invitrogen, Carlsbad, CA, USA) and 2\% BSA blocking solution (both for $30 \mathrm{~min}$ ) primary antibodies were applied overnight at $4{ }^{\circ} \mathrm{C}$ (goat anti-type I collagen, SouthernBiotech, Birmingham, UK; mouse collagen II Ab-3, Thermo Fisher Scientific, Waltham, MA, USA). Secondary antibodies were Alexa Fluor 488 donkey anti-goat and Alexa Fluor 488 goat anti-mouse (Molecular Probes, Eugene, OR, USA). Sections were counterstained with DAPI nuclear stain (Molecular Probes). Native articular cartilage and bone served as positive controls; negative controls were performed by omitting the primary antibody.

Statistical analysis. All samples were assayed by real-time PCR in triplicates. The values were log transformed and reported as the mean \pm SD of the real-time PCR analyses. The differentiation index was calculated as the ratio of the Col2 expression (mean) divided by the Coll expression (mean).

\section{Results}

Expression of Coll and Col2. During the six weeks of monolayer culture we observed a $>1000$-fold decrease in Col2 expression compared to native cartilage. The cultivation in HYAFF-11 as well as in Bio-Gide led in turn to an increase in Col2 expression, although with a different rate and extent. Whereas in HYAFF-11, the Col2 expression increased rather continuously to 16-fold, the Col2 expression in Bio-Gide remained unaltered until day 24 , thereafter increasing to 78 -fold in the last two weeks (Fig. 1a).

Coll expression increased a thousand times in the monolayer culture compared to the native cartilage. However, cultivation in both scaffolds caused an immediate and continuous decline ( $\sim 10$-fold) in Col1 expression (Fig. 1b).

The calculated differentiation index rose between 60 -fold (in Bio-Gide) and 470-fold (in HYAFF-11) after 38 days of $3 \mathrm{D}$ culture and demonstrated the increasing redifferentiation of the cells (Fig. 1c).

DAPI staining. A nuclear staining with DAPI was performed to illustrate cell distribution. After 3 days in Bio-Gide, a cell 

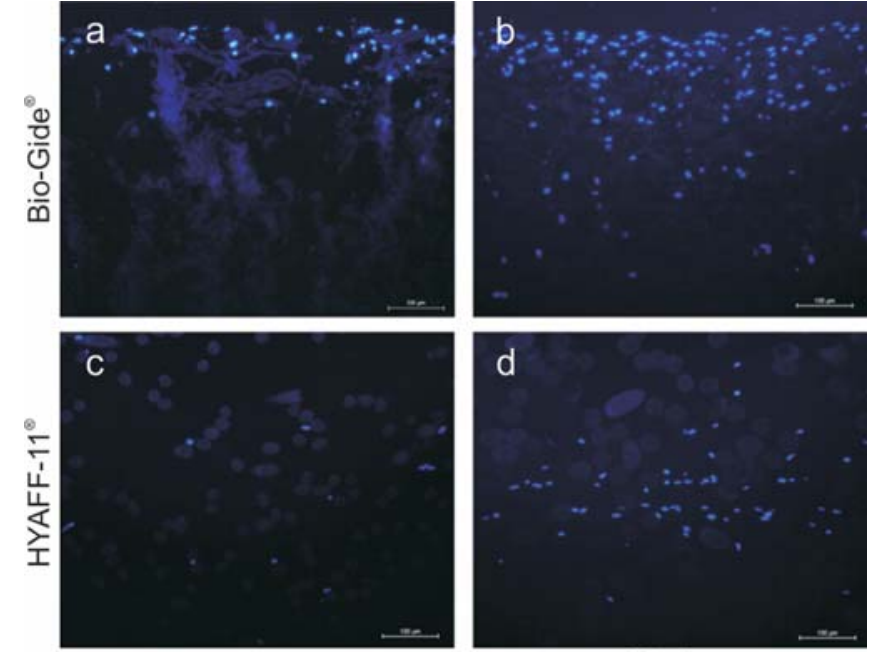

3 days

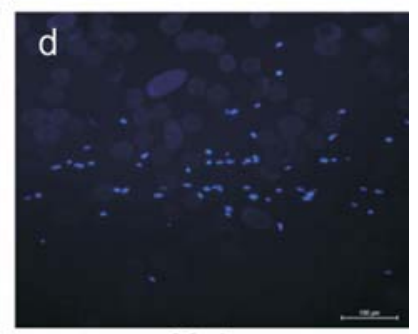

38 days

Figure 2. DAPI nuclear staining of dedifferentiated chondrocytes 3 and 38 days after seeding on a HYAFF-11 fleece or a Bio-Gide membrane. Scale bar, $100 \mu \mathrm{m}$.

distribution appeared that was restricted to the surface of the membrane (Fig. 2a). Five weeks later, we observed a higher cell density as well as several cells that had infiltrated deeper into the membrane (Fig. 2b).

Due to the loose structure of the HYAFF-11 fleece, the cells were evenly distributed over the whole scaffold from the beginning (Fig. 2c). After 38 days there was also a distinctly higher cell density (Fig. 2d).

Immunofluorescence. Col2 expression was detected in cells grown in the Bio-Gide membrane as well as the HYAFF-11 fleece. However, there was no recognizable difference between the different time points (Fig. 3a-d).
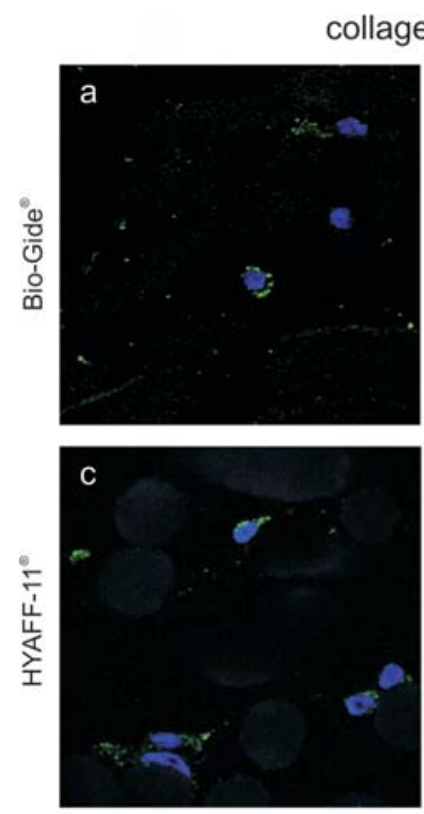

3 days
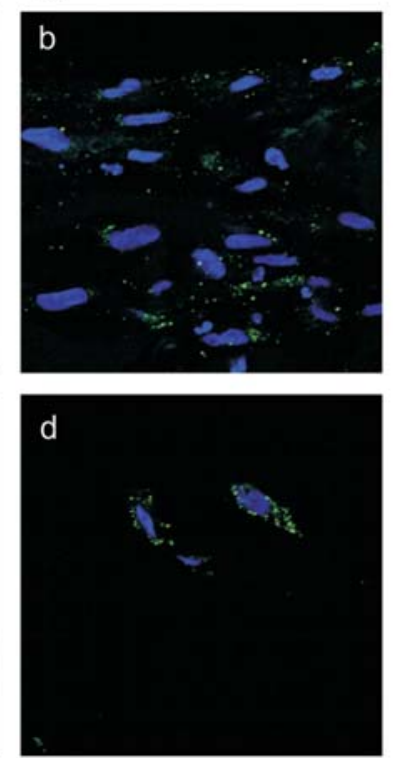

38 days
Although the Bio-Gide membrane consisted of Coll and therefore caused a strong background staining, some chondrocytes showed an intense Coll staining (Fig. 3e and f). After 38 days, especially, a large fraction of the cells exhibited weak or no Coll staining at all, whereas several cells were strongly positive for Col1 (Fig. 3f).

In the HAYFF fleece the chondrocytes showed a positive Coll staining as well, at both time points. In addition, there were membrane-like, Col1-positive structures visible on the bottom side of the fleece to which cells were attached (Fig. 3g and h).

BMP expression. GDF-5 expression was highly increased in the monolayer culture in contrast to the native cartilage (>100-fold). In the 3D culture, GDF-5 expression immediately decreased in both scaffolds to a level approximately 10-fold higher than in the native cartilage. During the following cultivation time, expression remained unchanged. In ligament fibroblasts, $G D F-5$ expression levels were between the levels of the native cartilage and that of the chondrocytes in the $3 \mathrm{D}$ culture (Fig. 4a).

In contrast to $G D F-5, B M P-2$ expression declined in the monolayer culture to $12.5 \%$ of the level of native cartilage. In the $3 \mathrm{D}$ culture a further decrease (one tenth) in the $B M P-2$ expression was observed (Fig. 4b).

$B M P-4$ expression showed characteristics very similar to $G D F-5$ expression. After a 100 -fold increase in the monolayer culture the expression decreased by a factor of ten during cultivation in the 3D culture (Fig. 4c).

$B M P-6$ expression resembled that of $B M P-2$. Similar to $B M P-2, B M P-6$ expression declined in the monolayer culture and further decreased in the 3D culture (Fig. 4d).

Noggin was dramatically down-regulated after six weeks of monolayer culture. At the beginning of the 3D culture, noggin expression stayed at the low level of chondrocytes in

Figure 3. Confocal laser micrographs of dedifferentiated chondrocytes 3 and 38 days after seeding on a HYAFF-11 fleece or a Bio-Gide membrane. Detection of Col2 and Col1 by IHC (Alexa 488 in green, DAPI nuclear staining in blue). 

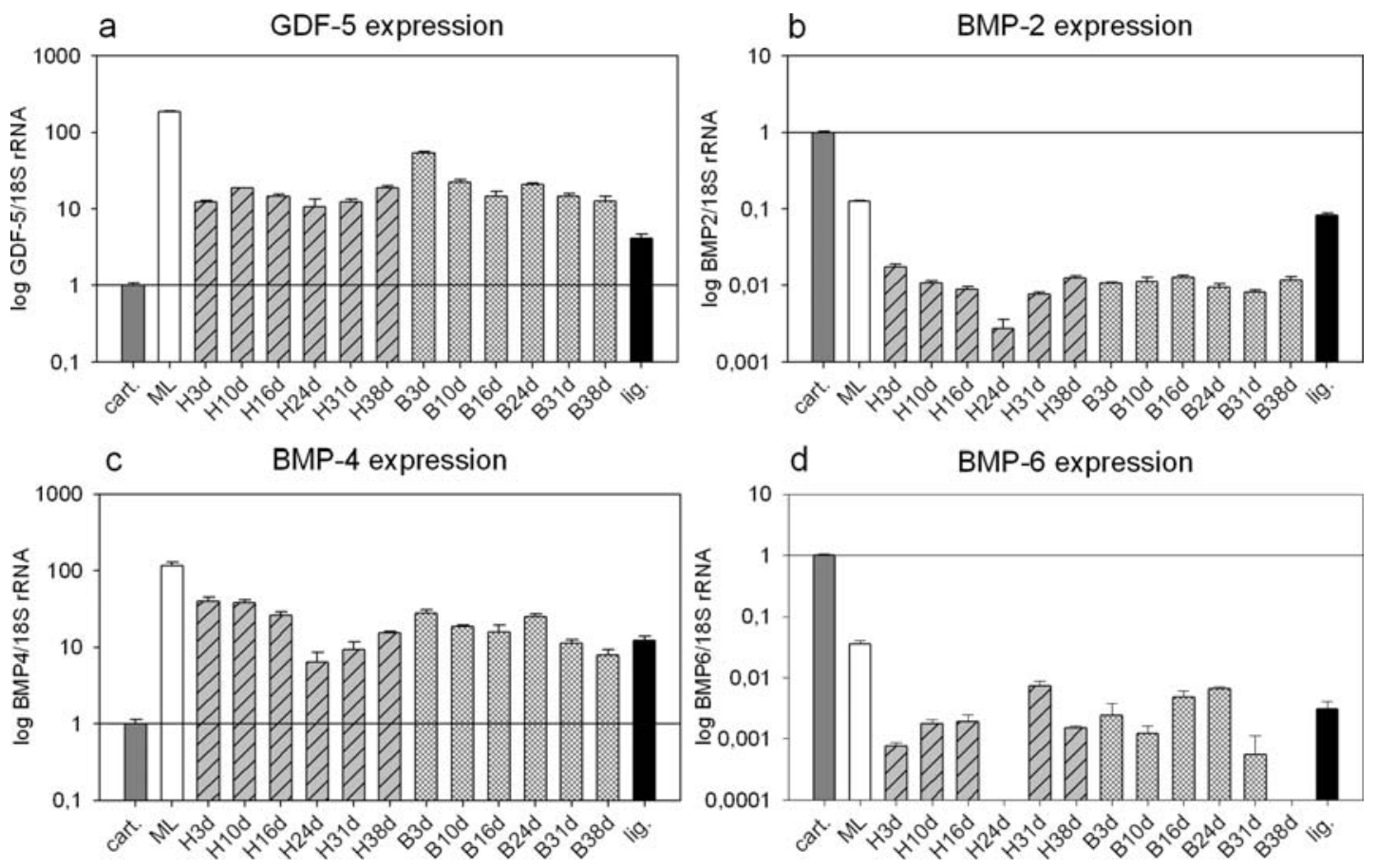

Figure 4. Real-time PCR analysis of BMP expression in native cartilage (cart.), dedifferentiated chondrocytes which were cultured for 6 weeks in monolayer (ML), dedifferentiated chondrocytes seeded on a HYAFF-11 fleece (H3d-H38d) or a Bio-Gide membrane and cultured for 3-38 days (B3d-B38d) and ligament fibroblasts (lig.). Gene expression was normalized to $18 S \mathrm{rRNA}$ expression. Data were log transformed and expressed as mean \pm SD of the real-time PCR triplicate analysis.
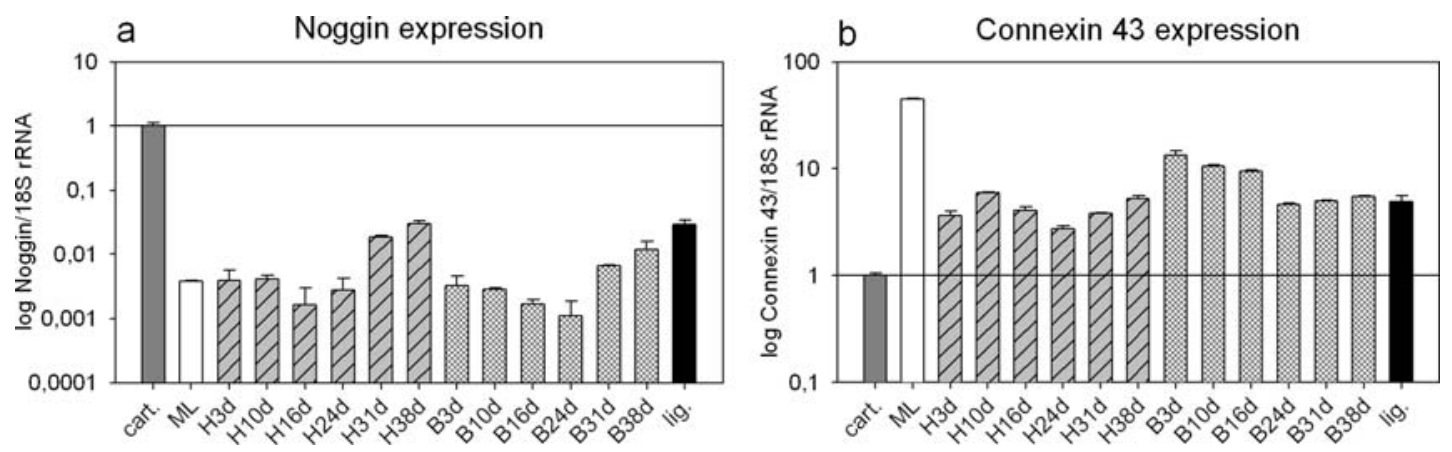

Figure 5. Real-time PCR analysis of noggin and connexin 43 expression in native cartilage (cart.), dedifferentiated chondrocytes which were cultured for 6 weeks in monolayer (ML), dedifferentiated chondrocytes seeded on a HYAFF-11 fleece (H3d-H38d) or a Bio-Gide membrane and cultured for 3-38 days (B3d-B38d) and ligament fibroblasts (lig.). Gene expression was normalized to $18 S \mathrm{r} R N A$ expression. Data were log transformed and expressed as mean $\pm \mathrm{SD}$ of real-time PCR triplicate analysis.

the monolayer culture. After 31 days, however, a slight increase in the noggin expression was detectable in both scaffolds (Fig. 5a).

Similar to $G D F-5$ expression, a higher expression of connexin 43 was detected after six weeks of monolayer culture. Chondrocytes in the 3D culture exhibited a slightly lower level of connexin 43 expression than in the monolayer culture, but was still higher when compared to native cartilage (Fig. 5b).

\section{Discussion}

One of the most common methods used to induce redifferentiation in chondrocytes is the $3 \mathrm{D}$ culture of cells on scaffolds. For this study we selected two frequently used scaffolds of completely different composition in order to analyse possible influences of the scaffolds on the redifferentiation behaviour of chondrocytes. One of the scaffolds was a fleece consisting of hyaluronic acid called HYAFF-11, the other a collagen type I/III membrane merchandised as Bio-Gide. Both scaffolds have been demonstrated to have a positive influence on the redifferentiation of chondrocytes (31). Furthermore, both scaffolds have been used for MACT for quite some time $(7,8)$.

By means of the differentiation index we were able to demonstrate a distinct tendency of cells in both scaffolds for redifferentiation during the cultivation in the $3 \mathrm{D}$ culture. The increase in the differentiation index was caused on the one 
hand by the decrease in the Coll expression at the first time point of analysis (day 3) and on the other hand by a slower rise in Col2 expression that exhibited scaffold-dependent differences. While Col2 expression began to increase in the HYAFF-11 fleece already at the beginning of the 3D culture, a rise in Col2 in the Bio-Gide membrane was not detectable until day 31 . However, this increase appeared more rapidly. These differences may have been caused by the unequal cell distribution in the scaffolds. In HYAFF-11, a relatively even distribution of the cells existed from the beginning of cultivation. In Bio-Gide, however, the cells were concentrated at the surface of the membrane. The closer contact between the cells therefore may have delayed the increase in Col2 expression until the time point when the cells were separated by the matrix produced and after some cells migrated into deeper layers of the membrane.

In search of novel approaches to improve the differentiation of chondrocytes, BMPs have been the object of interest in recent years. BMPs play an important role during the development of cartilage by regulating proliferation and differentiation of chondrocytes and their progenitors (32). Furthermore, they are of importance for the homeostasis of mature cartilage (33). This has led to numerous studies dealing with the influence of the addition and overexpression of BMPs on matrix synthesis of cultured chondrocytes. For a number of BMPs a positive effect on the synthesis of proteoglycans and collagens has been demonstrated $(30,34,35)$. Almost nothing, however, is known about the endogenous expression of BMPs in chondrocytes during cultivation and redifferentiation. Therefore, in order to obtain optimal redifferentiation conditions, knowledge of endogenous expression of BMPs is required for their application as external stimuli. For redifferentiation analysis we therefore focused on the expression of those genes which are most important for the development of cartilage.

Several BMPs were regulated quite differently, although between the two scaffolds only marginal differences were demonstrated. At the beginning of the 3D culture, GDF-5 and $B M P-4$ expression approached the values of the native cartilage through a distinct decrease. GDF-5 and BMP-4 are closely related proteins that are able to induce growth and differentiation of cartilage (36). In our case, the decrease in expression was associated with an induction of redifferentiation. Thus, it can be assumed that there is an association between the expression of both proteins and the proliferation rate of chondrocytes.

The function of GDF-5 depends on connexin 43 (37-39). Analogous to $G D F-5$ expression, there was a reduction in connexin 43 in the $3 \mathrm{D}$ culture. It was apparent that the decrease in GDF-5 and connexin 43 expression occurred more slowly in the Bio-Gide membrane than in HYAFF- 11. This could have been caused again by the different properties of the scaffolds. On the Bio-Gide membrane, chondrocytes formed a dense cell layer, and the cells were separated with time by the production of extracellular matrix.

Contrary to $G D F-5$ and $B M P-4$, which still show a relatively high expression in the $3 \mathrm{D}$ culture, $B M P-2$ and -6 expression dramatically decreased in the monolayer culture as well as in the $3 \mathrm{D}$ culture. $B M P-7$ was detected in neither sample.
If one assumes that redifferentiation imitates, at least partially, processes that happen during embryogenesis, then BMP-2, -4, -7 and GDF-5 may play an important role at the beginning of redifferentiation. Expression of BMP-6 is not generally demonstrated until later phases of cartilage development, particularly in hypertrophic chondrocytes (40). In context with our findings the question therefore arises, why only low or almost non-existent expression of $B M P-2,-6$ and -7 is observed. The cultivation on scaffolds seems to be an important stimulus for the induction of redifferentiation, but may not be sufficient to induce $B M P-2,-6$ or -7 expression. In contrast to cartilage development, important structures are missing in vitro, such as a perichondrium, an interzone or a synovial membrane. All of these exhibit important signal centres and express different BMPs $(40,41)$. In the absence of these signal centres an external stimulus may be necessary in order to obtain complete redifferentiation. Our data revealed that a combination of BMP-2, -6 and -7 may be promising, although the sequence, chronology and the extent of addition still have to be demonstrated.

Another factor that has to be addressed is the inhibitor noggin. It has been shown that noggin can inhibit BMPs by binding to them and preventing the interaction with their receptors (42). Since native cartilage expresses noggin, a balance between BMPs and noggin may be necessary to prevent the further differentiation towards hypertrophic chondrocytes and the calcification of the tissue. The reduction in noggin during redifferentiation therefore may amplify the effect of the added BMPs. For this reason, an optimally timed addition of noggin should be considered to avoid hypertrophy after redifferentiation. However, additional research must be undertaken to gain further insight into this complex field of delicately regulated system of growth and differentiation.

\section{Acknowledgements}

This study was supported by the Austrian Worker's Compensation (project 2/08).

\section{References}

1. Gelber AC, Hochberg MC, Mead LA, Wang NY, Wigley FM and Klag MJ: Joint injury in young adults and risk for subsequent knee and hip osteoarthritis. Ann Intern Med 133: 321-328, 2000.

2. Glowacki J, Trepman E and Folkman J: Cell shape and phenotypic expression in chondrocytes. Proc Soc Exp Biol Med 172: 93-98, 1983.

3. Schnabel M, Marlovits S, Eckhoff G, et al: Dedifferentiationassociated changes in morphology and gene expression in primary human articular chondrocytes in cell culture. Osteoarthritis Cartilage 10: 62-70, 2002.

4. Albrecht C, Schlegel W, Eckl P, et al: Alterations in CD44 isoforms and HAS expression in human articular chondrocytes during the de- and re-differentiation processes. Int J Mol Med 23: 253-259, 2009.

5. Marlovits S, Hombauer M, Tamandl D, Vecsei V and Schlegel W: Quantitative analysis of gene expression in human articular chondrocytes in monolayer culture. Int J Mol Med 13: 281-287, 2004.

6. Marlovits S, Hombauer M, Truppe M, Vecsei V and Schlegel W: Changes in the ratio of type-I and type-II collagen expression during monolayer culture of human chondrocytes. J Bone Joint Surg Br 86: 286-295, 2004.

7. Marlovits S, Zeller P, Singer P, Resinger C and Vecsei V: Cartilage repair: generations of autologous chondrocyte transplantation. Eur J Radiol 57: 24-31, 2006. 
8. Pavesio A, Abatangelo G, Borrione A, et al: Hyaluronan-based scaffolds (Hyalograft C) in the treatment of knee cartilage defects: preliminary clinical findings. Novartis Found Symp 249: 203-217; discussion 229-233, 234-208, 239-241, 2003.

9. Schneider U and Andereya S: First results of a prospective randomized clinical trial on traditional chondrocyte transplantation vs CaReS-Technology. Z Orthop Ihre Grenzgeb 141: 496-497, 2003.

10. Ochs BG, Muller-Horvat C, Rolauffs B, Fritz J, Weise K and Schewe B: Treatment of osteochondritis dissecans of the knee: one-step procedure with bone grafting and matrix-supported autologous chondrocyte transplantation. Z Orthop Unfall 145: 146-151, 2007

11. Chubinskaya S, Segalite D, Pikovsky D, Hakimiyan AA and Rueger DC: Effects induced by BMPS in cultures of human articular chondrocytes: comparative studies. Growth Factors 26: 275-283, 2008.

12. Kawanishi M, Oura A, Furukawa K, et al: Redifferentiation of dedifferentiated bovine articular chondrocytes enhanced by cyclic hydrostatic pressure under a gas-controlled system. Tissue Eng 13: 957-964, 2007.

13. Kurz B, Domm C, Jin M, Sellckau R and Schunke M: Tissue engineering of articular cartilage under the influence of collagen I/ III membranes and low oxygen tension. Tissue Eng 10: 1277-1286, 2004.

14. Chen D, Zhao M and Mundy GR: Bone morphogenetic proteins. Growth Factors 22: 233-241, 2004.

15. Massague J: TGF-beta signal transduction. Annu Rev Biochem 67: 753-791, 1998.

16. Zwijsen A, Verschueren K and Huylebroeck D: New intracellular components of bone morphogenetic protein/Smad signaling cascades. FEBS Lett 546: 133-139, 2003.

17. Kawabata M, Chytil A and Moses HL: Cloning of a novel type II serine/threonine kinase receptor through interaction with the type I transforming growth factor-beta receptor. J Biol Chem 270: 5625-5630, 1995 .

18. Koenig BB, Cook JS, Wolsing DH, et al: Characterization and cloning of a receptor for BMP-2 and BMP-4 from NIH 3T3 cells. Mol Cell Biol 14: 5961-5974, 1994.

19. Macias-Silva M, Hoodless PA, Tang SJ, Buchwald M and Wrana JL: Specific activation of Smad1 signaling pathways by the BMP7 type I receptor, ALK2. J Biol Chem 273: 25628-25636, 1998.

20. Rosenzweig BL, Imamura T, Okadome T, et al: Cloning and characterization of a human type II receptor for bone morphogenetic proteins. Proc Natl Acad Sci USA 92: 7632-7636, 1995.

21. Ten Dijke P, Yamashita H, Sampath TK, et al: Identification of type I receptors for osteogenic protein-1 and bone morphogenetic protein-4. J Biol Chem 269: 16985-16988, 1994.

22. Moustakas A and Heldin CH: From mono- to oligo-Smads: the heart of the matter in TGF-beta signal transduction. Genes Dev 16: 1867-1871, 2002.

23. Diekman BO, Rowland CR, Caplan AI, Lennon D and Guilak F: Chondrogenesis of adult stem cells from adipose tissue and bone marrow: Induction by growth factors and cartilage derived matrix. Tissue Eng Part A. Aug. 28, 2009 (Epub ahead of print).

24. Sekiya I, Larson BL, Vuoristo JT, Reger RL and Prockop DJ: Comparison of effect of BMP-2, -4 , and -6 on in vitro cartilage formation of human adult stem cells from bone marrow stroma. Cell Tissue Res 320: 269-276, 2005.

25. Steinert AF, Palmer GD, Pilapil C, Noth U, Evans CH and Ghivizzani SC: Enhanced in vitro chondrogenesis of primary mesenchymal stem cells by combined gene transfer. Tissue Eng Part A 15: 1127-1139, 2009.
26. Appel B, Baumer J, Eyrich D, et al: Synergistic effects of growth and differentiation factor-5 (GDF-5) and insulin on expanded chondrocytes in a 3-D environment. Osteoarthritis Cartilage 17: 1503-1512, 2009.

27. Bobacz K, Gruber R, Soleiman A, Erlacher L, Smolen JS and Graninger WB: Expression of bone morphogenetic protein 6 in healthy and osteoarthritic human articular chondrocytes and stimulation of matrix synthesis in vitro. Arthritis Rheum 48: 2501-2508, 2003.

28. Che JH, Zhang ZR, Li GZ, Tan WH, Bai XD and Qu FJ: Application of tissue-engineered cartilage with BMP-7 gene to repair knee joint cartilage injury in rabbits. Knee Surg Sports Traumatol Arthrosc 18: 496-503, 2010.

29. Hautier A, Salentey V, Aubert-Foucher E, et al: Bone morphogenetic protein-2 stimulates chondrogenic expression in human nasal chondrocytes expanded in vitro. Growth Factors 26: 201-211, 2008.

30. Lin L, Zhou C, Wei X, et al: Articular cartilage repair using dedifferentiated articular chondrocytes and bone morphogenetic protein 4 in a rabbit model of articular cartilage defects. Arthritis Rheum 58: 1067-1075, 2008.

31. Schlegel W, Nurnberger S, Hombauer M, Albrecht C, Vecsei V and Marlovits S: Scaffold-dependent differentiation of human articular chondrocytes. Int J Mol Med 22: 691-699, 2008.

32. Enomoto-Iwamoto M, Iwamoto M, Mukudai Y, et al: Bone morphogenetic protein signaling is required for maintenance of differentiated phenotype, control of proliferation, and hypertrophy in chondrocytes. J Cell Biol 140: 409-418, 1998.

33. Lories RJ and Luyten FP: Bone morphogenetic protein signaling in joint homeostasis and disease. Cytokine Growth Factor Rev 16: 287-298, 2005.

34. Chubinskaya S, Hakimiyan AA, Rappoport L, Yanke A, Rueger DC and Cole BJ: Response of human chondrocytes prepared for autologous implantation to growth factors. J Knee Surg 21: 192-199, 2008.

35. Yamaoka H, Asato H, Ogasawara T, et al: Cartilage tissue engineering using human auricular chondrocytes embedded in different hydrogel materials. J Biomed Mater Res A 78: 1-11, 2006.

36. Hatakeyama Y, Tuan RS and Shum L: Distinct functions of BMP4 and GDF5 in the regulation of chondrogenesis. J Cell Biochem 91: 1204-1217, 2004.

37. Chatterjee B, Meyer RA, Loredo GA, Coleman CM, Tuan R and Lo CW: BMP regulation of the mouse connexin43 promoter in osteoblastic cells and embryos. Cell Commun Adhes 10: 37-50, 2003.

38. Coleman CM, Loredo GA, Lo CW and Tuan RS: Correlation of GDF5 and connexin 43 mRNA expression during embryonic development. Anat Rec A Discov Mol Cell Evol Biol 275: 1117-1121, 2003.

39. Schlegel W, Albrecht C, Eckl P, et al: Dedifferentiation of human articular chondrocytes is associated with alterations in expression patterns of GDF-5 and its receptors. J Cell Mol Med 13: 3398-3404, 2009.

40. Xiao YT, Xiang LX and Shao JZ: Bone morphogenetic protein. Biochem Biophys Res Commun 362: 550-553, 2007.

41. Goldring MB, Tsuchimochi K and Ijiri K: The control of chondrogenesis. J Cell Biochem 97: 33-44, 2006.

42. Tsumaki N, Nakase T, Miyaji T, et al: Bone morphogenetic protein signals are required for cartilage formation and differently regulate joint development during skeletogenesis. J Bone Miner Res 17: 898-906, 2002. 